\title{
ABEDUlares DE LA SiERRA DE SAN MAMEDE (ORENSE, ESPAÑA)
}

\author{
Margarita COSTA, Juan HIGUERAS \& Carlos MORLA
}

\begin{abstract}
RESUMEN: En el presente trabajo se aborda el estudio de las formaciones de abedul en el límite meridional de la región galaica. Se describe su estructura, composición florística y se establece su dinamismo tanto progresivo como regresivo. Asi mismo se propone su inclusión en la asociación Luzulo henriquesii-Betuletum celtibericae Rivas Martínez 1964. Complementariamente se aportan datos sobre su situación actual y se plantean diversas alternativas para su conservación.
\end{abstract}

Palabras clave: Abedul, conservación, Galicia, España.

\begin{abstract}
In this paper a study of the birch-tree forest along the south limit of the "Galaica" region is done. Their structure and floristic composition is described. It is appointed both their progresive and degradating dynamism. It is also suggested the inclusion of these forest in the phytological association Luzulo henriquesii-Betuletum celtibericae Rivas Martínez 1964. Their actual situation is commented too and several proceeding for their conservation are suggested.
\end{abstract}

Key words: Birch-tree, conservation, Galicia, Spain,

\section{N T R O D U C C I O N}

Los abedulares de la Sierra de "San Mamede", bosques residuales de las masas de caducifolios que se extendieron por gran parte del Macizo Central de Orense, constituyen, junto con los de las lucenses sierras de Ancares y Caurel, uno de los bosques mejor conservados de Galicia. Se localizan en el término municipal de Montederramo, concretamente en los montes denominados de "San Mamede" y "O Teixedo" (cuadro de vértices UTM: PG 2377, PG 2677 y PG 2674).

Se desarrollan sobre amplias laderas de pendiente media o elevada (30-80\%) sobre sustratos de pizarras, cuarcitas o granitos.

No disponemos de datos climáticos precisos pero en las estaciones más próxims se recogen precipitaciones totales relativamente elevadas (Cabeza de Manzaneda $2252 \mathrm{~mm}$ y Camba $2037 \mathrm{~mm}$ (Morla, 1983). El régimen hídrico se ve favorecido además por su orientación, siempre en exposiciones de umbría. 
Además de constituir una singularidad paisajística en un marco territorial dominado por brezales y cultivos, hay que destacar el interés que tienen estos bosques por representar uno de los enclaves de vegetación eurosiberiana en situación más avanzada hacia el mundo vegetal mediterráneo en el noroeste peninsular. En el resto de los macizos orensanos las manifestaciones de abedul se restringen a las condiciones favorables de vaguadas o áreas de afloramientos de acuíferos. Desde el punto de vista faunístico se constituyen en uno de los biotopos más representativos del macizo.

\section{F IS O N O M I Y ESTR U C T URA}

En las mejores manifestaciones del bosque, el abedul o "bidueiro" (Betula celtiberica Rothm. \& Vasc.) es el dominante en el estrato arbóreo; con una altura media de 8-12 m no son raros los árboles de más de $20 \mathrm{~m}$. Generalmente los pies de abedul proceden de brotes de cepa o chirpiales con una media de 6-8 por cepa, aunque en algún caso hayamos contado 18-20. En lugares poco accesibles se pueden encontrar viejos ejemplares constituidos por una gran cepa de 2-3 m de diámetro y 1-2 de altura, de la que emergen 4 ó 5 gruesos tallos, conjuntamente con numerosas huellas de antiguos troncos eliminados por talas, práctica común en estos montes. Los portes en candelabro también pueden tener un origen natural; los viejos troncos abatidos por el viento o la nieve suelen producir abundantes renuevos gracias a la gran capacidad de esta especie para regenerarse vegetativamente. La vitalidad de las cepas debe mantenerse durante mucho tiempo, pues es común encontrar en el seno del bosque ejemplares con muestras de varias generaciones de recepes y a la vez con un numeroso grupo de brotes jóvenes. No obstante, también aparecen dispersas grandes y viejas cepas semiagotadas, que sustentan pies con muy poca vitalidad, atacados frecuentemente por Piptoporus betulinus (Dull.: Fr.) P. Karst. El desarrollo de los renuevos se produce con gran rapidez, tanto en situaciones despejadas como bajo cubierta del propio bosque.

Las condiciones de luminosidad necesarias para la producción de brinzales de abedul son inexistentes en el interior del bosque asi como en las repoblaciones de Pinus sylvestris L. que se extienden por la sierra. Sin embargo bajo la cubierta del abedular y del pinar sí se aprecia regereración de "cancireixo" o serbal de cazadores (Sorbus aucuparia L.) y de acebo (Ilex aquifolium L.). Ambos táxones conforman un estrato arbóreo inferior aunque el acebo, en algunos casos, se manifiesta en rodales monoespecíficos con pies de hasta $14 \mathrm{~m}$ de altura.

En este territorio el bosque de abedules se desarrolla con gran vitalidad y óptimo estado vegetativo en interfluvios y situaciones de ladera (orientaciones $\mathrm{N}$ y NE), siempre por encima de los $1200 \mathrm{~m}$. Por debajo de esta cota $B$. celtiberica se va a limitar progresivamente a las vaguadas y bordes de cauces, situación generalizada de esta especie en áreas más orientales y meridionales orensanas (Bellos, 1966).

El tapiz herbáceo bajo el abedular es muy denso y continuo. Las especies dominantes en el mismo son Luzula sylvatica (Hudson) Gaudin subsp. henriquesii (Degen) P. Silva y Deschampsia flexuosa (L.) Trin.; la segunda predomina donde la humedad es menor. Si la iluminación es mayor y no hay encharcamiento se desarro- 
lla con profusión Anthoxanthum amarum Boiss.

En las vaguadas y proximidades de cauces aparece la "abelaira" o avellano (Corylus avellana L.) que puede llegar a hacerse dominante. Asimismo se hacen patentes otros táxones exigentes en humedad como Ranunculus platanifolius L., Allium victorialis L., Chrysosplenium oppositifolium L., etc.

Bajo el punto de vista corológico destaca el elevado porcentaje de táxones endémicos iberoatlánticos, euatlánticos y eurosiberianos presentes en estas formaciones, algunos de los cuales encuentran aquí el límite de su avance hacia el interior peninsular como Daboecia cantabrica (Hudson) C. Koch y Ulex gallii Planchon subsp. breoganii (Castroviejo \& Valdés Bermejo) Rivas Martínez, Diaz, F. Prieto, Loidi \& Penas. Otro aspecto notable de estos abedulares bajo el punto de vista florístico, es la presencia de Poligonatum verticillatum (L.) All., taxon prácticamente ausente en el resto del macizo de "Manzaneda-Queixa-San Mamede".

\section{CONS IDER A C I ON ES D I N A I C A S}

En la Sierra de "San Mamede" los bosques naturales ocupan una pequeña proporción de su superficie, aproximadamente 220 Has (Fig. 1).

El paisaje vegetal presenta una estructura en mosaico donde se intercalan las distintas etapas de sustitución propias de sus series evolutivas. En el caso del abedular, el primer nivel de leñosas seriales está integrado por un brezal-tojal de talla baja con Ulex gallii subsp. breoganii, Daboecia cantabrica, Halimium alysoides (Lam.) C. Koch, Calluna vulgaris (L.) Hull, Erica cinerea L. Vaccinium myrtillus L., Chamaespartium tridentatum (L.) P. Gibbs, Lithodora diffusa (Lag.) I.M. Johnston, etc. En fase algo más avanzada se incorpora Erica australis L. subsp. aragonensis (Willk.) P. Cout., proporcionándole mayor talla y biomasa al brezal. La siguiente etapa es la "xesteira" o piornal, dominado por Genista florida L. subsp. polygaliphylla (Brot.) P. Cout.; esta fase se instala directamente en todos aquellos terrenos que han sido cultivados o destinados a prados de siega, y cuyo abandono ha sido lugar común en los dos últimos decenios.

Los primeros árboles que aparecen son ya "bidueiros" y "cancireixos"; estos táxones heliófilos comparten la dominancia en los primeros años, aunque con el tiempo la mayor talla de $B$. celtiberica hace que éste permanezca finalmente como hegemónico en el paisaje. La proporción de uno u otro taxon en estas etapas inciales es bastente variable y depende de diveros factores. Así, en terrenos incendiados o removidos recientemente, donde la cubierta de matorral es escasa o nula, predomina generalmente $B$. celtiberica. Sin embargo, cuando el brezal ya está algo más desarrollado, la iluminación del suelo es menor y el abedul encuentra dificultades; es frecuente ver entonces como $S$. aucuparia se hace dominante o única especie arbórea de esa etapa colonizadora. La humedad del sustrato también juega un papel importante en este sentido favoreciendo al "bidueiro" frente al "cancireixo". Tampoco las zonas empradizadas son favorables para la regeneración del abedul; sin embargo sí se ven nutridos brinzales en las zonas trabajadas recientemente con maquinaria. 


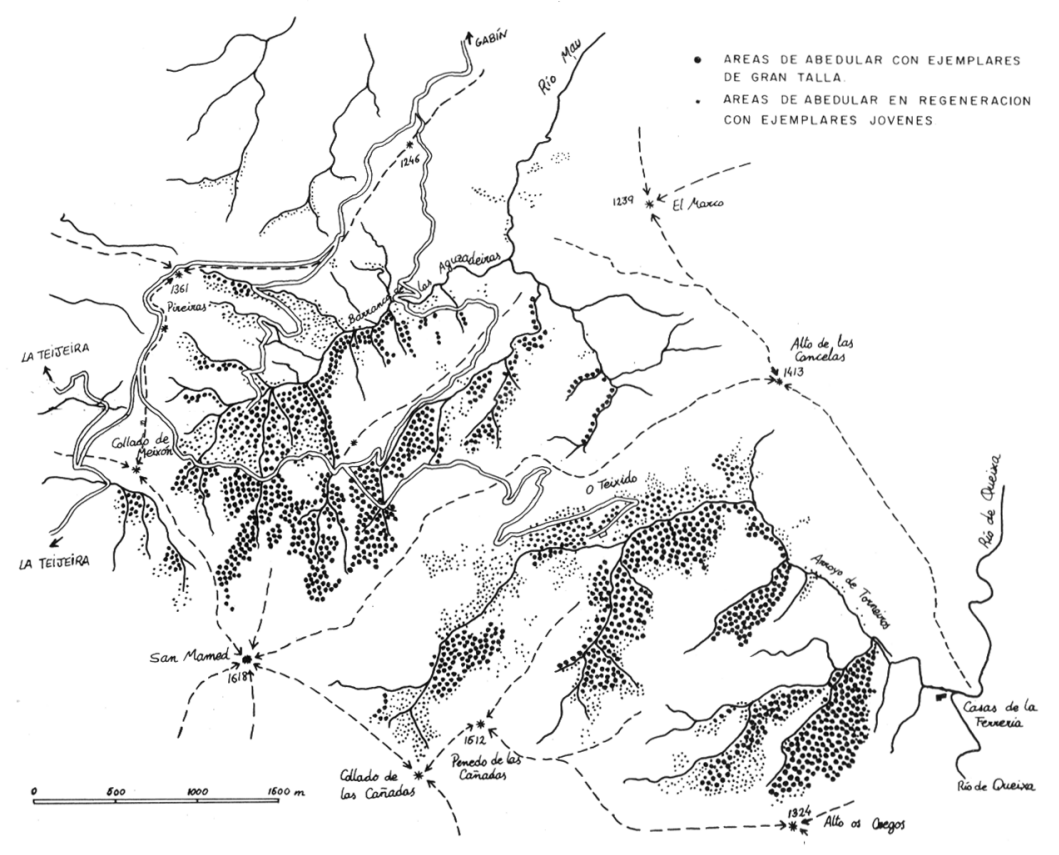

Figura 1.- Localización de los abedulares en la Sierra de San Mamede, con expresión de densidades y tamaño de los ejemplares.

El aspecto que consideramos más interesante bajo el punto de vista dinámico es la situación que se produce en el abedular con serbales ya desarrollado. La sombra no muy intensa de esta formación favorece la regeneración de Ilex aquifolium L. que poco a poco va conformando un sotobosque denso y pujante. El acebo también salpica el interior de las masas de Quercus robur L., pero el robledal en este área no suele superar los $1300 \mathrm{~m}$ mientras que el acebo sube más llegando a formar masas puras por encima del robledal (Fig. 2).

Los rodales de acebo se hacen cada vez mayores y aunque su talla no sobrepasa la de los abedules más altos, llega a formar extensos bosquetes que finalmente pueden suceder al abedular como formación terminal estable. En efecto, la estructura del acebo, su follaje denso y compacto, generan un ambiente extraordinariamente umbrófilo en el que abedules y serbales no son capaces de progresar. En esta situación, el tiempo juega a favor del acebo que puede seguir regenerándose tras la desaparición de los abedules y serbales. Paralelamente la composición florística varía, y del conjunto de táxones que componen el cortejo florístico del abedular (tabla 1) sólo permanecen aquellos capaces de soportar una prolongada y densa cubierta (Anemone nemorosa L., Oxalis acetosella L., Saxifraga spathularis Brot., et.). Este parece ser el origen de los acebales que salpican residualmente el macizo de "Manzaneda-QueixaSan Mamede", y que probablemente han estado mucho más desarrollados en tiempos pasados. (El acebo, además de regenerarse por semilla bajo su propia cubierta, presenta también una excelente capacidad para reproducirse vegetativamente, siendo frecuentes los ejemplares con numerosos brotes de cepa y raiz). 

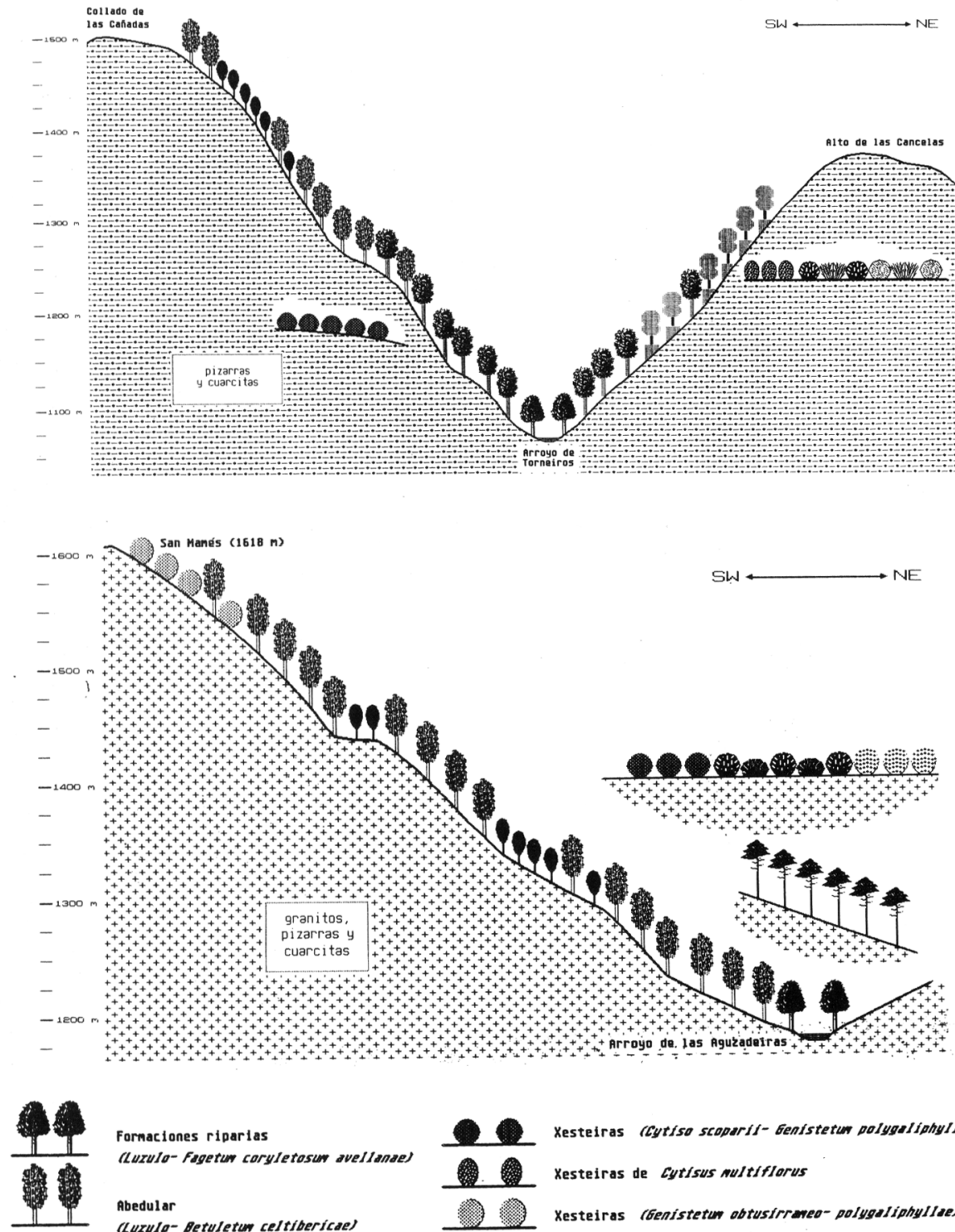

Formaciones riparias

(Lurujo-fagetum coryjetosum avellanae)

Abedular

(Lurula- Betuletur celtibericae)

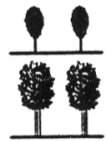

Acebeda

Robledal

1. Xesteiras (Cytiso scoparfi- Genistetur polygaliphyllides Xesteiras de Cytisus multiflorus

(Vaccinto- Duercetum roboris)

Rebollar

(Holco- Duercetum pyrensicde)

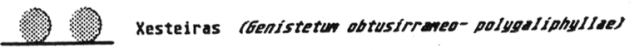

14. Brezal ipterosparto tridentati-Ericetur ardgonensis)

Whaly

stin Brezal Coboecio cantaticae- Erfcetur dragonensis)

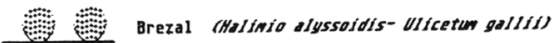

Repoblaciones de Pinus sylvestris

Figura 2.- Catenas representativas de la vegetación en la Sierra de San Mamede. 
Parece posible por consiguiente, que dinámicamente los abedulares que hoy encontramos puedan representar en muchos casos etapas seriales de acebedas potenciales. La acción antrópica sobre el monte favorece a los primeros, que en este piso altitudinal forman mosaicos con los distintos tipos y etapas de brezales, generados éstos por un nivel más acusado de explotación humana. Las acebedas, a causa de talas e incendios, han ido desapareciendo poco a poco; este proceso continúa en la actualidad y su posible recuperación se ve comprometida por la explotación a que se ven sometidos regularmente los abedulares.

Los bosquetes residuales de acebo sólo pueden encontrarse en algunos puntos muy alejados o poco accesibles del territorio que nos ocupa ("Val das Cañadas", "rio das Casas", "Os Bois",...).

El papel de los abedulares como primera etapa de sustitución de bosques más umbrófilos no se restringe a las acebedas. En esta misma sierra a los robledales montanos de Quercus robur, situados en niveles altitudinales inferiores, también se asocia el abedular con carácter serial. Y hemos observado que el mismo papel puede jugar en los rebollares ( $Q$. pyrenaica) húmedos de carácter más mediterráneo.

La progresión del bosque de acebos bajo la protección de otra formación forestal es extensible también a las repoblaciones de Pinus sylvestris de esta sierra. El mismo fenómeno puede observarse en otros puntos de la Península Ibérica como por ejemplo en los abedulares del Sistema Central o en los pinares de $P$. sylvestris del macizo del Moncayo (Ruiz de la Torre, 1984).

La estructura del bosque de acebos recuerda bastante a la de un hayedo. Son formaciones que dejan pasar muy poca luz siendo el sotobosque muy escaso, tanto en especies leñosas como herbáceas, con la diferencia de que en el caso de la acebeda el ambiente umbrófilo se prolonga todo el año. Estas particulares condiciones hacen que el cortejo florístico de las acebedas densas sea muy pobre, limitándose a un pequeño número de táxones de requerimientos ambientales compatibles. A escala peninsular sería interesante estudiar la relación entre el límite meridional de los hayedos y las manifestaciones de acebo en formación cerrada. Es precisamente en algunas de las áreas en que los hayedos se detienen ante la proximidad de los ambientes mediterráneos (Sistema Central, Sistema Ibérico Norte, Macizos Galaicos), donde las acebedas parecen representar una prolongación extrema del mundo vegetal eurosiberiano.

En efecto, el acebo, de espectro ambiental bastante amplio ${ }^{\star}$, puede aproximarse mucho más al régimen de veranos secos, si bien, al igual que falta Fagus sylvatica, están ausentes en su cortejo florístico otras especies eurosiberianas sensibles. La comparación de estos bosques con hayedos empobrecidos nos parece bastante acertada.

En el norte peninsular el límite del avance del haya hacia occidente coincide con la variación progresiva del régimen hídrico (Font Quer, 1954): el período seco estival se acentúa aunque el total de precipitaciones anuales pueda ser muy elevado; por otro lado, la disminución general de altitud de las montañas galaicas y su ramifi-

\footnotetext{
* Aisladamente el acebo alcanza las serranías béticas y si hay humedad suficiente puede llegar a integrarse en formaciones de encinar (Ruiz de la Torre, 1984)
} 
cación van haciendo desaparecer poco a poco el efecto de barrera que para las influencias mediterráneas meridionales ejercen las perfectas alineaciones cantábricas. Al contrario que el Sistema Ibérico, donde testimonios fósiles y referencias antiguas indican que Fagus sylvatica alcanzó latitudes más meridionales que en la actualidad, en territorio gallego no existe ningún indicio de que el haya pudiera haber ocupado en el pasado posiciones al occidente de su límite actual.

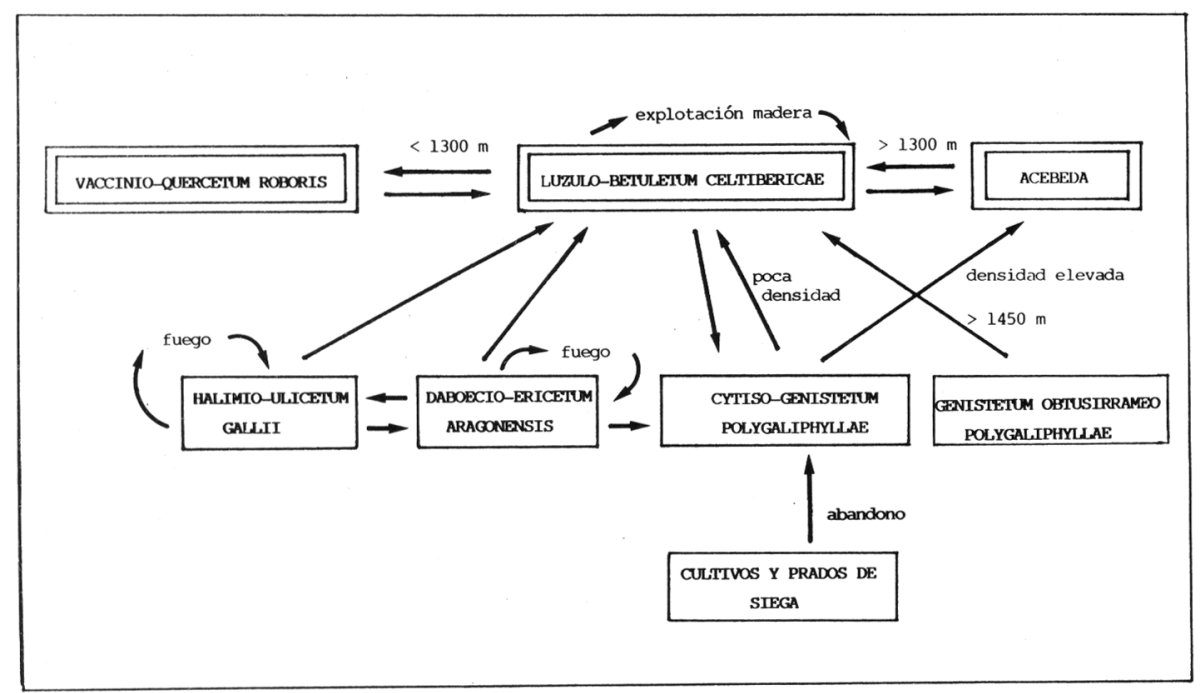

Representación de las relaciones dinámicas entre las relaciones principales formaciones arbustivas y arbóreas en la Sierra de San Mamede.

\section{P OS I CION F I T OS OCIOLOG I CA}

Fitosociológicamente los abedulares de la Sierra de "San Mamede", en el subsector juresiano-queixense, sector gallego-portugués, de la provincia Cántabro-Atlántica (Rivas Martínez, 1988), no han sido objeto de ningún estudio particular.

En el ya clásico trabajo sobre la vegetación de Galicia (Bellot, 1966), se hacen escasas referencias a las formaciones de abedul de las zonas meridionales galaicas y lo más concreto que se puede leer es el siguiente comentario: "... muchas zonas altitudinales gallegas llevan variantes caracterizadas por la presencia del abedul en un elevado índice ... Pueden ser consideradas estas zonas como facies Betuletosum roborisGallaecicum Bellot 1949".

Posteriormente, Castroviejo (1977) comenta respecto a los abedulares de la Sierra del Invernadeiro que "serían incluibles en la asociación Luzulo-Betuletum celtibericae Rivas Martínez 1964". 
En los últimos años se han realizado algunos trabajos sintéticos sobre la vegetación de la Península Ibérica o de amplias zonas de la misma próximas al territorio que nos ocupa (Peinado Lorca \& Rivas Martínez, 1987; Rivas Martínez, 1988; Rivas Martínez \& col., 1984). Ciñéndonos a los esquemas sintaxonómicos propuestos recientemente nos encontramos con dos tipos de abedulares en el noroeste peninsular: Luzulo henriquesii-Betuletum celtibericae Rivas Martínez 1964 y Saxifrago spathularidis-Betuletum celtibericae Rivas Martínez 1981. El primero, claramente eurosiberiano, caracteriza el piso altimontano orocantábrico sobre sustratos silíceos y parece alcanzar su límite suroriental en las sierras gallegas de Ancares y Caurel en la provincia de Lugo. Sin embargo este límite está confuso en la literatura. Izco \& col. (1985) comentan la mencionada asociación en la Sierra del Caurel y admiten que la misma había sido citada también en la Sierra del Invernadeiro. Los mismos autores posteriormente (1986) establecen claramente el límite suroriental del Luzulo-Betuletum en la lucense Sierra del Caurel indicando expresamente que la adscripción de los abedulares del "Invernadeiro" al Luzulo-Betuletum "ha de revocarse". Finalmente Izco (1987) representa esta asociación en el macizo de "Queixa" aunque no aporta justificación en el texto.

El segundo tipo presenta ya un matiz mediterráneo que se pone de manifiesto por la ausencia en su cortejo florístico de determinados táxones atlánticos y sobre todo por las etapas seriales que lo acampañan. Son abedulares característicos del piso altimontano galaico-portugués y del supramediterráneo orensano-sanabriense y estrellense sobre sustratos silíceos. Es en este tipo en el que se incluyen más recientemente las formaciones juresiano-queixenses objeto de este trabajo (Rivas Martínez, 1988).

Por nuestra parte hemos realizado algunos inventarios en los abedulares de los montes de "San Mamede" y "O Teixedo" con los que hemos elaborado la tabla 1. Cotejándola con las proporcionadas por otros autores de algunas zonas galaicas, orocantábricas y estrellenses (Rivas Martínez \& col., 1984; Izco \& col., 1986; Fernández Prieto \& M. Vazquez, 1987; Rivas Martínez, 1981), consideramos que dichos abedulares deben incluirse definitivamente en la asociación Luzulo henriquesiiBetuletum celtibericae Rivas Martínez 1964. Las etapas de sustitución muestran asimismo el carácter eurosiberiano de estas comunidades tal como hemos comentado en el apartado anterior. "Xesteiras" o piornales de la asociación Cytiso scoparii-Genistetum polygaliphyllae Rivas Matínez, Diaz, F. Prieto, Loidi \& Penas 1984 en primer lugar, seguidas, en fases de degradación más avanzadas, por brezales de Daboecio cantabricae-Ericetum aragonensis Rivas Martínez, in F. Prieto \& Loidi 1984 y Halimio alyssoidis-Ulicetum gallii (Rivas Martínez 1979) Rivas Martínez, Diaz, F. Prieto, Loidi \& Penas 1984, avalan dicha consideración.

\section{DATOS HISTORICOS Y S ITUACIONACTUAL}

Tanto de la lectura e interpretación del paisaje en que se enmarcan las formaciones de abedul de la Sierra de "San Mamede" como de los datos históricos del área, se evidencia que nunca dispusieron de algún tipo de protección que los sustrajera del devenir del medio. 
Desde la Edad Media con propiedad señorial hasta varios siglos despues en régimen foral del Monaterio de Montederramo, el monte en el que se enclavan mantuvo asignación vecinal de carácter abierto, con aprovechamientos de leñas, pastoreo y roza de matorral que, en distintas circunstancias coyunturales se intensificaron con apropiaciones de parcelas, permanentes o temporales, para prados y cultivos.

Ha sido, por lo tanto, la difícil accesibilidad hasta fechas muy recientes, la localización en las cotas más elevadas (entre 1200 y $1550 \mathrm{~m}$ ) y mayor pendiente, y el alejamiento de los asentamientos poblacionales, la casuística que ha evitado, en nuestro criterio, su degradación irreversible por cortas. Así como las condiciones de humedad edáfica y ambiental y de estructura las que permitieron su resistencia a fuegos inmemoriales.

Según nuestras observaciones y datos obtenidos directamente de los paisanos, estas dos acciones antropógenas, sostenidas tradicionalmente hasta la actualidad, han tenido utilidades y manifestaciones diversas. Los pies de Quercus robur ("carballo") y de Q. pyrenaica ("rebolo"), además de cubrir las necesidades constructivas rurales y del propio Monasterio, suministraron leña pra hogares, hornos e incluso fundición artesanal de hierro, complementadas por especies de estratos inferiores como Genista florida subsp. polygaliphylla (piorno) o cepas de Erica australis subsp. aragonensis "(torgos)". Los mangos de herramientas y postes de cerramientos eran de Ilex aquifolium. La corteza del acebo de uso medicinal, se recogió para su venta hasta finales de los años treinta. La madera de Betula celtiberica ("bido") se ha empleado secularmente para elaborar los elementos de carros y aperos. Además, las quemas extensivas y periódicas no sólo fueron utilizadas para eliminar la cubierta leñosa y mantener la regresión vegetal para el pastoreo, sino que, a favor de condiciones morfológicas, cumplieron también la función dinamizadora de arrastre de nutrientes a los prados localizados en zonas inferiores.

Estas últimas prácticas quedaron excluidas a partir de 1947 en aquellos terrenos del monte "Sierra de San Mamede" en que la Administración Forestal inició repoblaciones con Pinus sylvestris en las que el fuego reinició su presencia coyuntural hace unos 10 años, arruinando una parte del pinar.

Las repoblaciones que se extendieron a casi las dos terceras partes del monte ocupando zonas con facies degradadas, no produjeron efectos edáficos negativos por la preparación manual del terreno, ni incidieron en el abedular a excepción de los bordes inferiores en que se evidencian diferencias de temperamento interespecífico reforzadas por la intervención inicial.

Las extracciones puntuales a las que permanentemente se sometió el abedular, se sistematizaron por la Administración Forestal en el período 1958-67 (véase la tabla 2) con una corta generalizada a gran parte de la masa que, acentuando una estructura de monte bajo, produjo unas subastas de 16.099 apeas de abedul que ubicaron $252 \mathrm{~m}^{3}$.

Al considerar la problemática actual de estos bosques se constituye en indispensable la referencia al medio humano directamente relacionado con ellos. Sus características socioeconómicas son las generales de los territorios gallegos de la montaña interior, en concreto del Macizo Central, y se corresponden con los parámetros de una economía agraria muy deprimida. 


\begin{tabular}{crrrr} 
№ corta & Año & $\begin{array}{c}\text { № apeas } \\
1-2,5 \mathrm{~m}\end{array}$ & $\begin{array}{r}\text { Volumen m3 c/c } \\
\text { Diámetro máximo } \\
\text { de las apeas (cm) }\end{array}$ \\
\hline 1 & 1958 & 2796 & 124,30 & 50 \\
2 & 1959 & 2401 & 113,23 & - \\
3 & 1961 & 644 & 37,56 & - \\
4 & 1962 & 1655 & 78,89 & - \\
5 & 1963 & 2130 & 111,31 & - \\
6 & 1964 & 161 & 14,24 & - \\
7 & 1966 & 3549 & 209,12 & 54 \\
8 & 1967 & 2763 & 163,05 &
\end{tabular}

Tabla 2. Aprovechamientos de madera de abedul en el monte de San Mamede en el período 1958-67. Fuente: Servicio de Producción Forestal

Los 130 habitantes de la comunidad ${ }^{\star}$, que detentan la propiedad en mano común del monte, se dispersan en ocho núcleos del territorio parroquial, con una densidad notoriamente inferior a la media provincial. La pirámide poblacional es invertida, casi carente de elementos jóvenes. Asimismo disponen de un nivel precario de servicios e infraestructura.

Esta situación infiere una tendencia demográfica y económica fuertemente regresiva con retroceso notorio de la cabaña ganadera de vacuno, abandono de cultivos agrícolas (subsector por otra parte de vocación marginal) y desaparición inexorable de algunos asentamientos, como en breve se producirá con "A Magainza", localidad a pie de monte.

En este contexto las acciones perturbadoras de origen vecinal se concretan en:

Apetencias en su condición de titulares del monte, expresadas a la Administración Forestal, de aprovechamientos de abedules, ante la demanda sostenida de su madera.

Cortas puntuales incontroladas de acebo para estacas de cierres.

Riesgo de incendio en bordes del abedular por quemas esporádicas en parcelas de cultivo limítrofes a las zonas del monte en cotas inferiores. No obstante, es muy superior el peligro que implican las quemas períodicas en las fincas de propiedad privada, ubicadas al sur y oeste, a fín de controlar la evolución del matorral.

Efectos negativos en las regeneraciones del abedul, acebo y serbal del ganado vacuno que, ubicado en la finca "O Teixedo", pasta extensiva y estacionalmente en el monte.

Simultáneamente se producen actuaciones de particulares absentistas o de la propia Administración que interfieren la estabilidad de esta masa arbórea, con aten-

\footnotetext{
* Datos de elaboración propia a partir de "Rectificación del Padrón Municipal de habitantes a 1 de Enero de 1988".
} 
ción protectora nula por los primeros y reducida, en el segunda caso, al grado variable de voluntarismo de los sucesivos gestores forestales. Son significativas determinadas pistas trazadas arbitrariamente con efectos degradantes palpables y que se revelan o paradigmáticamente inútiles (en "O Teixedo" una de ellas se interrumpe por enfrentar un obstáculo no previsto) o contribuyen decisivamente a hipotecar el futuro del bosque, como es el caso de la que discurre por el borde inferior del abedular, construida por los Servicios Forestales hace tres años con el beneplácito vecinal.

La fauna experimenta una fuerte presión cinegética foránea, en ocasiones con prácticas simplificadoras y furtivismo añadido.

\section{PROPUESTAS DE CONSER A CION}

Se hace urgente la elaboración y aplicación por la Administración Autonómica, en cuanto que ejerce la tutela del monte vecinal y su gestión, de un programa específico que preserve al ecosistema abedular. Habrá de contemplar, al menos, los aspectos siguientes:

Conversión a propiedad pública tanto de la finca "O Teixedo" como de dos pequeños enclaves del monte vecinal, consiguiendo, complementariamente a una consolidación dominical imprescindible, eliminar uno de los focos de impactos negativos.

Ordenación del espacio monte, asignando usos selvícolas y ganaderos que, en todo caso, excluyan a la formación caducifolia.

En las reforestaciones que precisaran de una cierta diversificación de especies respecto al Pinus sylvestris, la utilización de Betula celtiberica facultará, conjuntamente con coníferas de crecimiento medio como Pseudotsuga menziesii, atender fines productivos en cotas inferiores.

Se paralizará la regresión de la ganadería vecinal con la mejora y creación de pastizales complementadas con diversas aportaciones infraestructurales como, entre otras, las sanitarias y divulgación y práctica de técnicas de ensilado y cultivos de forrajes.

Realización de aprovechamientos forestales, compensatorios y alternativos, mediante cortas en la masa de $P$. sylvestris, a hecho en los ecotonos del abedular facultando su expansión y restauración y de mejora por entresaca en el resto del pinar.

Ordenación cinegética que reserve el biotopo abedular, excluyendo interferencias sobre las poblaciones de vertebrados.

Con la dilación inherente, el plan a escala monte habrá de integrarse en otro global que posibilite la creación de un Espacio Natural Protegido que se extienda por gran parte del Macizo Central Orensano. El área, que abarcaría unas 23.000 Has distribuidas entre las Sierras de San Mamede y Queixa, Manzaneda y Montes de Invernadeiro, presenta peculiares valores geomorfológicos, botánicos, faunísticos, paisajísticos y culturales cuya protección debe armonizarse con el desarrollo socioeconómico de poblaciones marginadas. 
Con estos objetivos la única figura de posible aplicación entre las contenidas en la legislación vigente es la de Parque Natural u otra de nueva creación más ajustada. En la zonificación que asignara usos, al abedular le correspondería una protección total con dedicación a actividades educativas y científicas.

\section{B I B L I O G R A F I A}

BELLOT, F. -1966- La vegetación de Galicia. Anal. Inst. Bot. Cavanilles, 24:3-306. Madrid.

CARBAlLEIRA, A., DEVESA, C., RETUERTO, R., SANTILLAN, E. \& UCIEDA, F. 1983- Bioclimatología de Galicia. Fundación P. Barrié de la Maza. La Coruña.

CASTROVIEJO, S. -1977- Estudio sobre la vegetación de la aSierra del Invernadeiro (Orense). $M^{\circ}$ Agricultura. I.C.O.N.A., Madrid.

FERNANDEZ PRIETO, J.A. \& VAZQUEZ, V.M. -1987- Datos sobre los bosques asturianos orocantábricos occidentales. Lazaroa, 7:363-382. Madrid.

FONT QUER, P. -1954- La vegetación. In: M. Terán (Ed) "Geografía de España y Portugal, II": 143-271. Ed. Montaner y Simón. Barcelona.

IZCO, J., AMIGO, J. \& GUITIAN, J. -1986- Identificación y descripción de los bosques montanos del extremo occidental de la Cordillera Cantábrica. Trabajos Compostelanos de Biología, 13:183-202. Santiago de Compostela.

IZCO, J. -1987- "Galicia" in Peinado Lorca \& Rivas-Martínez eds. "La Vegetación de España" col.Aula Abierta Univ. Alcalá de Henares.

IZCO, J., AMIGO, J. \& GUITIAN, J. -1985- "Botánica" in Estudio del medio natural de las montañas gallegas. I. "O Caurel" Monografías de la Universidad de Santiago de compostela, 102:73-139.

MORLA, C. -1983- Estudio ecológico de la cubierta vegetal leñosa y análisis florístico en el Macizo Manzaneda-Queixa (Orense). Tesis Doctoral E.T.S. Ing. Montes. Madrid.

PEINADO LORCA, M. \& RIVAS MARTINEZ, S. (Eds.) -1987- La vegetación de España. Publ. Universidad de Alcalá de Henares.

RIVAS MARTINEZ, S. -1981- Sobre la vegtación de la Serra da Estrela (Portugal). Anal. Real Acad. Farmacia, 47(4):435-480. Madrid.

RIVAS MARTINEZ, S. -1988- Mapa de las series de vegetación de España (escala 1:400.000). Publ. I.C.O.N.A. Madrid.

RIVAS MARTINEZ, S., DIAZ, T.E., FERNANDEZ PRIETO, J.A., LOIDI, J. \& PENAS, A. -1984- La vegetación de la alta montaña cantábrica. Los Picos de Europa. Ed. Leonesas. León.

RUIZ DE LA TORRE, J. -1984- Las dehesas del vértice norte de Madrid. Montes, 1:8-14. Madrid.

(Aceptado para su publicación el 3 de marzo de 1990)

Dirección de los autores: M. Costa: Departamento de Biología Vegetal I. Facultad de Biología. Universidad Complutense de Madrid. J. HIGUERAS: Servicio de Producción Forestal. Consellería de Agricultura. Xunta de Galicia. Orense. C. MORLA: Cátedra de Botánica. Departamento de Silvopascicultura. E.T.S.I. Montes. Universidad Politécnica. Madrid. 
LUZULO HENRIQUESII-BETULETUM CELTIBERICAE Rivas-Martínez 1964 nom.mut.

(Ilici-Fagenion (Br.-Bl.1967) Rivas-Martínez 1973, Quercion robori-pyrenaicae (Br.-Bl., getea $\mathrm{Br} .-\mathrm{Bl}$. \& Vlieger in Vlieger 1937)

Altitud ( $1=10 \mathrm{~m})$
Inclinación $(\%)$
Orientación
Area $\left(\mathrm{m}^{2}\right)$
№ especies
№ de orden

Características y diferenciales de Asociación y Alianza:

Betula celtiberica Rothm. \& Vasc.

Luzula sylvatica (Hudson) Gaudin

subsp. henriquesii (Degen) P.Silva

Saxifraga spathularis Brot.

Vaccinium myrtillus L.

Deschampsia flexuosa (L.) Trin.

Blechnunt spicant (L.) Roth.

Polygonatum verticillatum (L.) All.

Melampyrum pratense L.

Características de Orden y Clase:

Sorbus aucuparia L.

Oxalis acetosella L.

Anemone nemorosa $\mathrm{L}$.

Ilex aquifolium L.

Poa nemoralis L.

Stellaria holostea L.

Dryopteris filix-mas (L.) Schott

Crepis lampsanoides (Gouan) Tausch

Compañeras:

Anthoxanthum amarum Boiss.

Polypodium vulgare L.

Erica arborea L.

Genista florida L. subsp. polygaliphylla

(Brot.) P.Cout.

Viola palustris L.

Corydalis claviculata (L.) DC. in Lam. \& DC.

Omphalodes nitida Hoffmans \& Link

$\begin{array}{lccc}130 & 125 & 135 & 134 \\ 50 & 50 & 30 & 60 \\ \mathrm{NE} & \mathrm{N} & \mathrm{NE} . & \mathrm{N} \\ 150 & 100 & 100 & 150 \\ 21 & 19 & 13 & 26 \\ 1 & 2 & 3 & 4\end{array}$

$\begin{array}{llll}4.4 & 4.4 & 3.3 & 3.3\end{array}$

$\begin{array}{llll}2.2 & 2.2 & 2.2 & 2.2\end{array}$

$\begin{array}{llll}1.2 & +.2 & 2.2 & 1.2\end{array}$

$\begin{array}{llll}2.2 & 1.2 & 2.2 & 2.2\end{array}$

$\begin{array}{llll}2.2 & 2.2 & 2.2 & 1.2\end{array}$

$+.2+.2$.

. +.2 . +

. +.2 . +

$\begin{array}{llll}2.2 & 2.2 & 2.2 & 2.2\end{array}$

$\begin{array}{llll}1.2 & +.2 & 1.2 & 1.2\end{array}$

$\begin{array}{llll}.2 & 1.2 & +.2 & 1.2\end{array}$

$1.2 \quad+.2+.2$

$\begin{array}{llll}2.2 & 1.2 & 2.2 \quad .\end{array}$

$+.2+.2+.2$

$+.2 \cdot+.2$

$+.2 \quad+\quad+$

$\begin{array}{cccc}2.2 & 2.2 & 2.2 & 2.2 \\ +.2 & +.2 & +.2 & +.2 \\ +.2 & . & . & +.2 \\ +.2 & . & . & +.2 \\ . & +.2 & . & + \\ +.2 & . & . & + \\ + & + & . & .\end{array}$

Ademas: Características de asociación y unidades superiores: Lonicera periclymenum L. + en 4; Euphorbia dulcis L. +.2 en l. Compañeras: Daboecia cantabrica (Hudson) C.Koch +.2 en 2; Digitalis purpurea L. +.2 en 2; Silene dioica (L.) Clairv. +.2, Salix caprea L. +.2 en l; Salix atrocinerea Brot. +.2, Lamium maculatum L. +, Caltha palustris L. +, Potentilla reptans L. +,Erythronium dens-canis L. +, Allium victorialis L. +, Narcissus triandrus L. +, Quercus robur L. + y Melica uniflora Retz + en 4.

Localidades: $\quad$ 1.- Monte San Mamede: Aguzadeiras

2.- Monte San Mamede: Penedo Negro

3.- Monte San Mamede: Las Garbias

4.- O Teixedo: San Giraldo 